\title{
THE FIRST SIMULTANEOUS SIGN CHANGE AND NON-VANISHING OF HECKE EIGENVALUES OF NEWFORMS
}

\author{
SANOLI GUN, BALESH KUMAR AND BIPLAB PAUL
}

\begin{abstract}
Let $f$ and $g$ be two distinct newforms which are normalized Hecke eigenforms of weights $k_{1}, k_{2} \geq 2$ and levels $N_{1}, N_{2} \geq 1$ respectively. Also let $a_{f}(n)$ and $a_{g}(n)$ be the $n$-th Fourier-coefficients of $f$ and $g$ respectively. In this article, we investigate the first sign change of the sequence $\left\{a_{f}\left(p^{\alpha}\right) a_{g}\left(p^{\alpha}\right)\right\}_{p^{\alpha} \in \mathbb{N}, \alpha \leq 2}$, where $p$ is a prime number. We further study the nonvanishing of the sequence $\left\{a_{f}(n) a_{g}(n)\right\}_{n \in \mathbb{N}}$ and derive bounds for first non-vanishing term in this sequence. We also show, using ideas of Kowalski-Robert-Wu and Murty-Murty, that there exists a set of primes $S$ of natural density one such that for any prime $p \in S$, the sequence $\left\{a_{f}\left(p^{n}\right) a_{g}\left(p^{m}\right)\right\}_{n, m \in \mathbb{N}}$ has no zero elements. This improves a recent work of Kumari and Ram Murty. Finally, using $\mathfrak{B}$-free numbers, we investigate simultaneous non-vanishing of coefficients of $m$-th symmetric power $L$-functions of non-CM forms in short intervals.
\end{abstract}

\section{INTRODUCTION}

For positive integers $k \geq 2, N \geq 1$, let $S_{k}(N)$ be the space of cusp forms of weight $k$ for the congruence subgroup $\Gamma_{0}(N)$ and $S_{k}^{\text {new }}(N)$ be the subspace of $S_{k}(N)$ consisting of newforms. We investigate arithmetic properties of Fourier-coefficients of $f \in S_{k}^{\text {new }}(N)$ which are normalized Hecke eigenforms. This question has been studied extensively by several mathematicians. In recent works, Kowalski, Lau, Soundararajan and $\mathrm{Wu}$ [15] and later Matomäki [22] showed that any $f \in S_{k}^{\text {new }}(N)$ which is a normalized Hecke eigenform is uniquely determined by the signs of their Hecke eigenvalues at primes. In this article, we investigate simultaneous sign change and non-vanishing of Hecke eigenvalues of such forms. More precisely, for $z \in \mathcal{H}:=\{z \in \mathbb{C}: \operatorname{Im}(z)>0\}, q:=e^{2 \pi i z}$, let

$$
f(z)=\sum_{n=1}^{\infty} a_{f}(n) q^{n} \in S_{k_{1}}^{n e w}\left(N_{1}\right) \quad \text { and } \quad g(z)=\sum_{n=1}^{\infty} a_{g}(n) q^{n} \in S_{k_{2}}^{\text {new }}\left(N_{2}\right)
$$

be two newforms which are normalized Hecke eigenforms. Here we study first sign change and non-vanishing of the sequence $\left\{a_{f}(n) a_{g}(n)\right\}_{n \in \mathbb{N}}$.

The question of simultaneous sign change for arbitrary cusp forms was first studied by Kohnen and Sengupta [14] under certain conditions which were later removed by the first

2010 Mathematics Subject Classification. 11F30, 11F11.

Key words and phrases. newforms, first simultaneous sign change, simultaneous non-vanishing, Rankin-Selberg method, $\mathfrak{B}$-free numbers. 
author, Kohnen and Rath [9]. In the later paper, the authors prove infinitely many sign change of the sequence $\left\{a_{f}(n) a_{g}(n)\right\}_{n \in \mathbb{N}}$. Here we prove the following theorem.

Theorem 1. Let $N_{1}, N_{2}$ be square-free, $N:=\operatorname{lcm}\left[N_{1}, N_{2}\right]$ and $f \in S_{k_{1}}^{\text {new }}\left(N_{1}\right), g \in S_{k_{2}}^{\text {new }}\left(N_{2}\right)$ be two distinct normalized Hecke eigenforms with Fourier expansions as in (1). Then there exists a prime power $p^{\alpha}$ with $\alpha \leq 2$ and

$$
p^{\alpha} \ll_{\epsilon} \max \left\{\exp \left(c \log ^{2}(\sqrt{\mathfrak{q}(f)}+\sqrt{\mathfrak{q}(g)})\right),\left[N^{2}\left(1+\frac{k_{2}-k_{1}}{2}\right)\left(\frac{k_{1}+k_{2}}{2}\right)\right]^{1+\epsilon}\right\}
$$

such that $a_{f}\left(p^{\alpha}\right) a_{g}\left(p^{\alpha}\right)<0$. Here $c>0$ is an absolute constant and $\mathfrak{q}(f), \mathfrak{q}(g)$ are analytic conductors of Rankin-Selberg $L$-functions of $f$ and $g$ respectively.

We use Rankin-Selberg method and an idea of Iwaniec, Kohnen and Sengupta [12] to prove Theorem 1. This theorem can be thought of as a variant of Strum's result about distinguishing two newforms by their Fourier-coefficients. This result can be compared with the results of Lau-Liu-Wu [19], Kohnen [13], Kowalski-Michel-Vanderkam [16], Ram Murty [25] and Sengupta [32].

Next we investigate sign changes of the sequence $\left\{a_{f}(n) a_{g}\left(n^{2}\right)\right\}_{n \in \mathbb{N}}$ in short intervals. This question of sign change for the sequence $\left\{a_{f}(n) a_{g}(n)\right\}_{n \in \mathbb{N}}$ in short intervals was considered by Kumari and Ram Murty (see [18, Theorem 1.6]). Here we prove the following.

Theorem 2. Let

$$
f(z)=\sum_{n=1}^{\infty} a_{f}(n) q^{n} \in S_{k_{1}}^{\text {new }}\left(N_{1}\right) \quad \text { and } \quad g(z)=\sum_{n=1}^{\infty} a_{g}(n) q^{n} \in S_{k_{2}}^{\text {new }}\left(N_{2}\right)
$$

be two distinct normalized Hecke eigenforms. For any sufficiently large $x$ and any $\delta>\frac{17}{18}$, the sequence $\left\{a_{f}(n) a_{g}\left(n^{2}\right)\right\}_{n \in \mathbb{N}}$ has at least one sign change in $\left(x, x+x^{\delta}\right]$. In particular, the number of sign changes for $n \leq x$ is $\gg x^{1-\delta}$.

Sign changes of Hecke eigenvalues implies non-vanishing of Hecke eigenvalues. The question of non-vanishing of Hecke eigenvalues has been studied by several mathematicians. One of the fundamental open problem in this area is a question of Lehmer which predicts that $\tau(n) \neq 0$ for all $n \in \mathbb{N}$, where $\tau(n)$ is the Ramanujan's $\tau$-function defined as follows;

$$
\Delta(z):=\sum_{n=1}^{\infty} \tau(n) q^{n}:=q \prod_{n=1}^{\infty}\left(1-q^{n}\right)^{24} .
$$

It is well known that $\Delta(z) \in S_{12}(1)$ is the unique normalized Hecke eigenform. We now investigate non-vanishing of the sequence $\left\{a_{f}\left(p^{m}\right) a_{g}\left(p^{m}\right)\right\}_{m \in \mathbb{N}}$ and our first theorem in this direction is the following. 
Theorem 3. Let

$$
f(z)=\sum_{n=1}^{\infty} a_{f}(n) q^{n} \in S_{k_{1}}^{\text {new }}\left(N_{1}\right) \quad \text { and } \quad g(z)=\sum_{n=1}^{\infty} a_{g}(n) q^{n} \in S_{k_{2}}^{\text {new }}\left(N_{2}\right)
$$

be two distinct normalized Hecke eigenforms. Then for all primes $p$ with $\left(p, N_{1} N_{2}\right)=1$, the set

$$
\left\{m \in \mathbb{N} \mid a_{f}\left(p^{m}\right) a_{g}\left(p^{m}\right) \neq 0\right\}
$$

has positive density.

The first author along with Kohnen and Rath (see Theorem 3 of [9]) showed that for infinitely many primes $p$, the sequence $\mathcal{A}_{p}:=\left\{a_{f}\left(p^{m}\right) a_{g}\left(p^{m}\right)\right\}_{m \in \mathbb{N}}$ has infinitely many sign changes and hence in particular, $\mathcal{A}_{p}$ has infinitely many non-zero elements. Theorem 3 shows that for all primes $p$ with $\left(p, N_{1} N_{2}\right)=1$, the non-zero elements of the sequence $\mathcal{A}_{p}$ has positive density and hence does not follow from Theorem 3 of [9]. Our next theorem strengthens Theorem 1.2 of Kumari and Ram Murty [18].

Theorem 4. Let

$$
f(z)=\sum_{n=1}^{\infty} a_{f}(n) q^{n} \in S_{k_{1}}^{n e w}\left(N_{1}\right) \quad \text { and } \quad g(z)=\sum_{n=1}^{\infty} a_{g}(n) q^{n} \in S_{k_{2}}^{\text {new }}\left(N_{2}\right)
$$

be two distinct normalized non-CM Hecke eigenforms. Then there exists a set $S$ of primes with natural density one such that for any $p \in S$ and integers $m, m^{\prime} \geq 1$, we have

$$
a_{f}\left(p^{m}\right) a_{g}\left(p^{m^{\prime}}\right) \neq 0 \text {. }
$$

Now we shall consider the question of the first simultaneous non-vanishing which is analogous to the question considered in Theorem 1. Our result here is as follows.

Theorem 5. Let

$$
f(z)=\sum_{n=1}^{\infty} a_{f}(n) q^{n} \in S_{k_{1}}\left(N_{1}\right) \quad \text { and } \quad g(z)=\sum_{n=1}^{\infty} a_{g}(n) q^{n} \in S_{k_{2}}\left(N_{2}\right)
$$

be two distinct normalized Hecke eigenforms. Further assume that $N:=\operatorname{lcm}\left[N_{1}, N_{2}\right]>12$. Then there exists a positive integer $1<n \leq(2 \log N)^{4}$ with $(n, N)=1$ such that

$$
a_{f}(n) a_{g}(n) \neq 0 \text {. }
$$

Further, when $N$ is odd, then there exists an integer $1<n \leq 16$ with $(n, N)=1$ such that

$$
a_{f}(n) a_{g}(n) \neq 0 .
$$

Note that $a_{f}(1) a_{g}(1)=1 \neq 0$ but we are trying to find the first natural number $n>1$ with $(n, N)=1$ for which $a_{f}(n) a_{g}(n) \neq 0$ which we call the first non-trivial simultaneous nonvanishing. Though first simultaneous sign change (see [19], also Theorem[1]above) implies first 
non-trivial simultaneous non-vanishing but the bound proved in Theorem 5 is much stronger for first non-trivial simultaneous non-vanishing.

The paper is organized as follows. In the next section, we introduce notations and briefly recall some preliminaries. In sections 3 to 7 , we provide proofs of theorems mentioned in the introduction. Finally, in the last section, using $\mathfrak{B}$-free numbers, we deduce certain results about simultaneous non-vanishing of coefficients of symmetric power $L$-functions of non-CM forms in short intervals.

\section{NOTATION AND PRELIMINARIES}

Throughout the paper, $p$ denotes a prime number and $\mathcal{P}$ denotes the set of all primes. We say that a subset $S \subset \mathcal{P}$ has natural density $d(S)$ if the limit

$$
\lim _{x \rightarrow \infty} \frac{\#\{p \in \mathcal{P}: p \leq x \text { and } p \in S\}}{\#\{p \in \mathcal{P}: p \leq x\}}
$$

exists and equal to $d(S)$. For any non-negative real number $x$, we denote the greatest integer $n \leq x$ by $[x]$. Let $A$ be a subset of the set of natural numbers. Then we say the density of the set $A$ is $d(A)$ if the limit

$$
\lim _{x \rightarrow \infty} \frac{\#\{n \leq x: n \in A\}}{\#\{n \leq x\}}
$$

exists and equal to the real number $d(A)$. For any $n, m \in \mathbb{N}$, we shall denote the greatest common divisor of $n$ and $m$ by $(n, m)$.

For a normalized Hecke eigenform $f \in S_{k}^{\text {new }}(N)$ with Fourier expansion

$$
f(z)=\sum_{n \geq 1} a_{f}(n) q^{n}
$$

we write

$$
\lambda_{f}(n):=\frac{a_{f}(n)}{n^{(k-1) / 2}} .
$$

From the theory of Hecke operators, we know

$$
\lambda_{f}(1)=1 \quad \text { and } \quad \lambda_{f}(m) \lambda_{f}(n)=\sum_{d \mid(m, n),(d, N)=1} \lambda_{f}\left(\frac{m n}{d^{2}}\right) .
$$

Also by a celebrated work of Deligne, we have

$$
\left|\lambda_{f}(n)\right| \leq d(n) \text { for all }(n, N)=1,
$$

where $d(n)$ denotes the number of positive divisors of $n$.

The following result of Kowalski-Robert-Wu [17, Lemma 2.3] (see also Murty-Murty [26. Lemma 2.5] ) plays an important role in the proof of Theorem 4 , 
Lemma 6. Let

$$
f(z):=\sum_{n=1}^{\infty} a_{f}(n) q^{n} \in S_{k}^{\text {new }}(N)
$$

be a normalized non-CM Hecke eigenform. For $\nu \geq 1$, let

$$
P_{f, \nu}:=\left\{p \in \mathcal{P} \mid p \nmid N \text { and } \lambda_{f}\left(p^{\nu}\right)=0\right\} .
$$

Then for any $\nu \geq 1$, we have

$$
\#\left(P_{f, \nu} \cap[1, x]\right) \ll_{f, \delta} \frac{x}{(\log x)^{1+\delta}}
$$

for any $x \geq 2$ and $0<\delta<1 / 2$. Here the implied constant depends on $f$ and $\delta$. Let

$$
P_{f}:=\cup_{\nu \in \mathbb{N}} P_{f, \nu}
$$

Then for any $x \geq 2$ and $0<\delta<1 / 2$, we have

$$
\#\left(P_{f} \cap[1, x]\right) \ll_{f, \delta} \frac{x}{(\log x)^{1+\delta}},
$$

where the implied constant depends only on $f$ and $\delta$.

We now recall some well known properties of Rankin-Selberg $L$-function associated with $f \in S_{k_{1}}^{\text {new }}\left(N_{1}\right)$ and $g \in S_{k_{2}}^{\text {new }}\left(N_{2}\right)$ which are normalized Hecke eigenforms. Suppose that $k_{1} \leq k_{2}$. One can now define the Rankin-Selberg $L$-function as follows

$$
R(f, g ; s):=\sum_{n \geq 1} \lambda_{f}(n) \lambda_{g}(n) n^{-s},
$$

which is absolutely convergent for $\Re(s)>1$ and hence it defines a holomorphic function there. Let $M:=\operatorname{gcd}\left(N_{1}, N_{2}\right)$ and $N:=\operatorname{lcm}\left[N_{1}, N_{2}\right]$ be square-free. By the work of Rankin [29] (see also [27], page 304), one knows that the function $\zeta_{N}(2 s) R(f, g ; s)$ is entire if $f \neq g$, where $\zeta_{N}(s)$ is defined by

$$
\zeta_{N}(s):=\prod_{p \nmid N}\left(1-p^{-s}\right)^{-1} \quad \text { for } \Re(s)>1 .
$$

We also have the completed Rankin-Selberg $L$-function

(6) $\quad R^{*}(f, g ; s):=(2 \pi)^{-2 s} \Gamma\left(s+\frac{k_{2}-k_{1}}{2}\right) \Gamma\left(s+\frac{k_{1}+k_{2}}{2}-1\right) \prod_{p \mid M}\left(1-c_{p} p^{-s}\right)^{-1} \zeta_{N}(2 s) R(f, g ; s)$

with $c_{p}= \pm 1$ depending on the forms $f$ and $g$. It is well known by the works of Ogg (see [27. Theorem 6]) and Winnie Li (see [20, Theorem 2.2]) that the completed Rankin-Selberg $L$ function satisfies the functional equation

$$
R^{*}(f, g ; s)=N^{1-2 s} R^{*}(f, g ; 1-s) .
$$




\section{Proof of TheOREM 1}

Throughout this section, we assume that $N_{1}$ and $N_{2}$ are square-free and $f \in S_{k_{1}}^{\text {new }}\left(N_{1}\right)$, $g \in S_{k_{2}}^{\text {new }}\left(N_{2}\right)$ are two distinct normalized Hecke eigenforms with $1<k_{1} \leq k_{2}$. In order to prove Theorem 1, we need to prove the following Propositions.

Proposition 7. For square-free integers $N_{1}, N_{2}$, let $f \in S_{k_{1}}^{\text {new }}\left(N_{1}\right), g \in S_{k_{2}}^{\text {new }}\left(N_{2}\right)$ be normalized Hecke eigenforms with $f \neq g$ and let $N:=\operatorname{lcm}\left[N_{1}, N_{2}\right]$ and $M:=\left(N_{1}, N_{2}\right)$. Then for any $t \in \mathbb{R}$ and $\epsilon>0$, one has

$$
\begin{aligned}
& \zeta_{N}(2+2 \epsilon+2 i t) R(f, g ; 1+\epsilon+i t) \ll_{\epsilon} 1 \\
& \text { and } \quad \zeta_{N}(-2 \epsilon+2 i t) R(f, g ;-\epsilon+i t) \ll_{\epsilon} N^{2+4 \epsilon}\left(1+\frac{k_{2}-k_{1}}{2}\right)^{1+2 \epsilon}\left(\frac{k_{1}+k_{2}}{2}\right)^{1+2 \epsilon}|1+i t|^{2(1+2 \epsilon)},
\end{aligned}
$$

where $\zeta_{N}(s)$ is defined in (5).

Proof. Since $\zeta_{N}(2+2 \epsilon+2 i t)$ and $R(f, g ; 1+\epsilon+i t)$ are absolutely convergent for $\Re(s)>1$, we have the first inequality. To derive the second inequality, we use functional equation. From the functional equation (7), we have

$$
\begin{aligned}
\zeta_{N}(2-2 s) \cdot R(f, g ; 1-s)= & (2 \pi)^{2-4 s} \cdot N^{2 s-1} \cdot \frac{\Gamma\left(s+\frac{k_{2}-k_{1}}{2}\right)}{\Gamma\left(1-s+\frac{k_{2}-k_{1}}{2}\right)} \cdot \frac{\Gamma\left(s+\frac{k_{1}+k_{2}}{2}-1\right)}{\Gamma\left(-s+\frac{k_{1}+k_{2}}{2}\right)} \\
& \cdot \prod_{p \mid M}\left(\frac{1-c_{p} p^{s-1}}{1-c_{p} p^{-s}}\right) \cdot \zeta_{N}(2 s) \cdot R(f, g ; s) .
\end{aligned}
$$

Using Stirling's formula (see page 57 of [10]), we have

$$
\text { and }\left|\frac{\Gamma\left(\frac{k_{1}+k_{2}}{2}+\epsilon+i t\right)}{\Gamma\left(\frac{k_{1}+k_{2}}{2}-1-\epsilon+i t\right)}\right| \ll_{\epsilon}\left(\frac{k_{1}+k_{2}}{2}\right)^{1+2 \epsilon}|1+i t|^{1+2 \epsilon} .
$$

For all $t \in \mathbb{R}$, we also have

$$
\begin{aligned}
& \left|\prod_{p \mid M}\left(1-c_{p} p^{-1-\epsilon-i t}\right)^{-1}\right|=\left|\prod_{p \mid M} \sum_{m \geq 0}\left(c_{p} p^{-1-\epsilon-i t}\right)^{m}\right| \leq \prod_{p \mid M} \sum_{m \geq 0}\left(p^{-1-\epsilon}\right)^{m} \ll_{\epsilon} 1 \\
& \text { and }\left|\prod_{p \mid M}\left(1-c_{p} p^{\epsilon+i t}\right)\right|=\prod_{p \mid M}\left|1-c_{p} p^{\epsilon+i t}\right| \leq \prod_{p \mid M}\left(1+p^{\epsilon}\right) \leq \prod_{p \mid M} p^{1+\epsilon} \ll_{\epsilon} M^{1+2 \epsilon} .
\end{aligned}
$$

Putting $s=1+\epsilon+i t$ in (8) and using the above estimates along with the first inequality, we get the second inequality.

The next proposition provides convexity bound for Rankin-Selberg $L$-function $R(f, g ; s)$. 
Proposition 8. For square-free integers $N_{1}, N_{2}$, let $f \in S_{k_{1}}^{\text {new }}\left(N_{1}\right), g \in S_{k_{2}}^{\text {new }}\left(N_{2}\right)$ be normalized Hecke eigenforms with $f \neq g$ and $N:=\operatorname{lcm}\left[N_{1}, N_{2}\right]$. Then for any $t \in \mathbb{R}, \epsilon>0$ and $1 / 2<\sigma<1$, one has

$$
R(f, g ; \sigma+i t) \ll_{\epsilon} N^{2(1-\sigma+\epsilon)}\left(1+\frac{k_{2}-k_{1}}{2}\right)^{1-\sigma+\epsilon}\left(\frac{k_{1}+k_{2}}{2}\right)^{1-\sigma+\epsilon}(3+|t|)^{2(1-\sigma)+\epsilon} .
$$

To prove this proposition, we shall use the following strong convexity principle due to Rademacher.

Proposition 9 (Rademacher [28]). Let $g(s)$ be continuous on the closed strip $a \leq \sigma \leq b$, holomorphic and of finite order on $a<\sigma<b$. Further suppose that

$$
|g(a+i t)| \leq E|P+a+i t|^{\alpha}, \quad|g(b+i t)| \leq F|P+b+i t|^{\beta}
$$

where $E, F$ are positive constants and $P, \alpha, \beta$ are real constants that satisfy

$$
P+a>0, \quad \alpha \geq \beta .
$$

Then for all $a<\sigma<b$ and for all $t \in \mathbb{R}$, we have

$$
|g(\sigma+i t)| \leq\left(E|P+\sigma+i t|^{\alpha}\right)^{\frac{b-\sigma}{b-a}}\left(F|P+\sigma+i t|^{\beta}\right)^{\frac{\sigma-a}{b-a}} .
$$

We are now ready to prove Proposition 8 .

Proof. We apply Proposition 9 with

$$
\begin{aligned}
a & =-\epsilon, \quad b=P=1+\epsilon, \quad F=C_{2}, \\
E & =C_{1} N^{2+4 \epsilon}\left(1+\frac{k_{2}-k_{1}}{2}\right)^{1+2 \epsilon}\left(\frac{k_{1}+k_{2}}{2}\right)^{1+2 \epsilon}, \alpha=2+4 \epsilon, \beta=0,
\end{aligned}
$$

where $C_{1}, C_{2}$ are absolute constants depending only on $\epsilon$. Thus for any $-\epsilon<\sigma<1+\epsilon$, we have

$\zeta_{N}(2 \sigma+2 i t) R(f, g ; \sigma+i t) \ll_{\epsilon}\left[N^{\frac{2+4 \epsilon}{1+2 \epsilon}}\left(1+\frac{k_{2}-k_{1}}{2}\right)\left(\frac{k_{1}+k_{2}}{2}\right)\right]^{1-\sigma+\epsilon}(1+\sigma+\epsilon+|t|)^{2(1-\sigma+\epsilon)}$.

Note that for $1 / 2<\sigma<1+\epsilon$, one knows

$$
\left|\zeta_{N}(2 \sigma+2 i t)\right|^{-1} \ll_{\epsilon} \log \log (N+2) \cdot|1+i t|^{\epsilon} .
$$

Combining all together, we get Proposition 8 .

As an immediate corollary, we have

Corollary 10. For square-free integers $N_{1}, N_{2}$, let $f \in S_{k_{1}}^{\text {new }}\left(N_{1}\right), g \in S_{k_{2}}^{\text {new }}\left(N_{2}\right)$ be normalized Hecke eigenforms with $f \neq g$ and $N:=\operatorname{lcm}\left[N_{1}, N_{2}\right]$. Then for any $t \in \mathbb{R}$ and any $\epsilon>0$, one has

$$
R(f, g ; 3 / 4+i t) \ll_{\epsilon}\left[N^{2}\left(1+\frac{k_{2}-k_{1}}{2}\right)\left(\frac{k_{1}+k_{2}}{2}\right)\right]^{1 / 4+\epsilon}(3+|t|)^{1 / 2+\epsilon} .
$$


Proposition 11. For square-free integers $N_{1}, N_{2}$, let $f \in S_{k_{1}}^{\text {new }}\left(N_{1}\right), g \in S_{k_{2}}^{\text {new }}\left(N_{2}\right)$ be normalized Hecke eigenforms with $f \neq g$ and $N:=\operatorname{lcm}\left[N_{1}, N_{2}\right]$. Then for any $\epsilon>0$, one has

$$
\sum_{\substack{n \leq x,(n, N)=1 \\ n \text { square-free }}} \lambda_{f}(n) \lambda_{g}(n) \log ^{2}(x / n) \ll_{\epsilon}\left[N^{2}\left(1+\frac{k_{2}-k_{1}}{2}\right)\left(\frac{k_{1}+k_{2}}{2}\right)\right]^{1 / 4+\epsilon} x^{3 / 4} .
$$

Proof. For any $\epsilon>0$, we know by Deligne's bound that

$$
\lambda_{f}(n) \lambda_{g}(n) \ll_{\epsilon} n^{\epsilon} .
$$

Hence by Perron's summation formula (see page 56 and page 67 of [24]), we have

$$
\sum_{\substack{n \leq x \\(n, N)=1 \\ n \text { square-free }}} \lambda_{f}(n) \lambda_{g}(n) \log ^{2}(x / n)=\frac{1}{\pi i} \int_{1+\epsilon-i \infty}^{1+\epsilon+i \infty} R^{b}(f, g ; s) \frac{x^{s}}{s^{3}} d s
$$

where

$$
R^{b}(f, g ; s)=\prod_{p \nmid N}\left(1+\frac{\lambda_{f}(p) \lambda_{g}(p)}{p^{s}}\right), \quad \Re(s)>1 .
$$

Further

$$
R(f, g ; s)=R^{b}(f, g ; s) H(s)
$$

where $H(s)$ has an Euler product which converges normally for $\Re(s)>1 / 2$. Now we shift the line of integration to $\Re(s)=3 / 4$. Observing that there are no singularities in the vertical strip bounded by the lines with $\Re(s)=1+\epsilon$ and $\Re(s)=3 / 4$ and using Proposition 8 along with (11), we have

$$
\sum_{\substack{n \leq x,(n, N)=1 \\ n \text { square-free }}} \lambda_{f}(n) \lambda_{g}(n) \log ^{2}(x / n)=\frac{1}{\pi i} \int_{3 / 4-i \infty}^{3 / 4+i \infty} R^{b}(f, g ; s) \frac{x^{s}}{s^{3}} d s .
$$

The above observations combined with Corollary 10 then implies that

$$
\sum_{\substack{n \leq x,(n, N)=1 \\ n \text { square-free }}} \lambda_{f}(n) \lambda_{g}(n) \log ^{2}(x / n) \ll_{\epsilon} N^{1 / 2+\epsilon}\left(1+\frac{k_{2}-k_{1}}{2}\right)^{1 / 4+\epsilon}\left(\frac{k_{1}+k_{2}}{2}\right)^{1 / 4+\epsilon} x^{3 / 4} .
$$

This completes the proof of the proposition.

Our next lemma will play a key role in proving Theorem 1.

Lemma 12. For square-free integers $N_{1}, N_{2}$, let $f \in S_{k_{1}}^{\text {new }}\left(N_{1}\right), g \in S_{k_{2}}^{\text {new }}\left(N_{2}\right)$ be normalized Hecke eigenforms with $f \neq g$ and $N:=\operatorname{lcm}\left[N_{1}, N_{2}\right]$. Also assume that for any $\alpha \leq 2, \lambda_{f}\left(p^{\alpha}\right) \lambda_{g}\left(p^{\alpha}\right) \geq 0$ for 
all $p^{\alpha} \leq x$. Then for $x \geq \exp \left(c \log ^{2}(\sqrt{\mathfrak{q}(f)}+\sqrt{\mathfrak{q}(g)})\right)$, we have

$$
\sum_{\substack{n \leq x,(n, N)=1 \\ n \text { square-free }}} \lambda_{f}(n) \lambda_{g}(n) \gg \frac{x}{\log ^{2} x} .
$$

Here $\mathfrak{q}(f), \mathfrak{q}(g)$ are analytic conductors of Rankin-Selberg L-functions of $f$ and $g$ respectively with

$$
\mathfrak{q}(f) \leq k_{1}^{2} N_{1}^{2} \log \log N_{1} \quad \text { and } \quad \mathfrak{q}(g) \leq k_{2}^{2} N_{2}^{2} \log \log N_{2}
$$

and $c>0$ is an absolute constant.

Proof. Using Hecke relation (3), for any prime $(p, N)=1$, we know that

$$
\lambda_{f}\left(p^{2}\right) \lambda_{g}\left(p^{2}\right)=\left[\lambda_{f}(p) \lambda_{g}(p)\right]^{2}-\lambda_{f}(p)^{2}-\lambda_{g}(p)^{2}+1 .
$$

By hypothesis $\lambda_{f}\left(p^{2}\right) \lambda_{g}\left(p^{2}\right) \geq 0$ for all $p \leq \sqrt{x}$. Hence for any $p \leq \sqrt{x}$ and $(p, N)=1$, we have

$$
\lambda_{f}(p)^{2} \lambda_{g}(p)^{2} \geq \lambda_{f}(p)^{2}+\lambda_{g}(p)^{2}-1 .
$$

This implies that

$$
\sum_{\substack{p \leq \sqrt{x},(p, N)=1}} \lambda_{f}(p)^{2} \lambda_{g}(p)^{2} \geq \sum_{\substack{p \leq \sqrt{x},(p, N)=1}} \lambda_{f}(p)^{2}+\sum_{\substack{p \leq \sqrt{x},(p, N)=1}} \lambda_{g}(p)^{2}-\sum_{\substack{p \leq \sqrt{x},(p, N)=1}} 1 .
$$

Using standard analytic techniques and prime number theorem for Rankin-Selberg $L$-functions of $f$ and $g$ respectively (see [11], pages 94-95, 110-111 for further details), we see that

$$
\sum_{\substack{p \leq \sqrt{x},(p, N)=1}} \lambda_{f}(p)^{2} \lambda_{g}(p)^{2} \geq c_{1} \frac{\sqrt{x}}{\log x}
$$

provided $x \geq \exp \left(c \log ^{2}(\sqrt{\mathfrak{q}(f)}+\sqrt{\mathfrak{q}(g)})\right)$, where $c, c_{1}>0$ are absolute constants and $\mathfrak{q}(f), \mathfrak{q}(g)$ are as in equation (12). Using the hypothesis

$$
\lambda_{f}(p) \lambda_{g}(p) \geq 0 \quad \text { and } \quad \lambda_{f}\left(p^{2}\right) \lambda_{g}\left(p^{2}\right) \geq 0
$$

for all $p, p^{2} \leq x$ and assuming that $x \geq \exp \left(c \log ^{2}(\sqrt{\mathfrak{q}(f)}+\sqrt{\mathfrak{q}(g)})\right)$, we have

$$
\begin{aligned}
\sum_{\substack{n \leq x,(n, N)=1 \\
n \text { square-free }}} \lambda_{f}(n) \lambda_{g}(n) & \geq \frac{1}{2} \sum_{\substack{p, q \leq \sqrt{x},(p q, N)=1, p \neq q}} \lambda_{f}(p q) \lambda_{g}(p q) \\
& =\frac{1}{2}\left(\sum_{\substack{p \leq \sqrt{x},(p, N)=1}} \lambda_{f}(p) \lambda_{g}(p)\right)^{2}-\frac{1}{2} \sum_{\substack{p \leq \sqrt{x},(p, N)=1}} \lambda_{f}(p)^{2} \lambda_{g}(p)^{2} .
\end{aligned}
$$


Now using Deligne's bound, we get

$$
\begin{aligned}
\sum_{\substack{n \leq x,(n, N)=1 \\
n \text { square-free }}} \lambda_{f}(n) \lambda_{g}(n) & \geq \frac{1}{2}\left(\sum_{\substack{p \leq \sqrt{x},(p, N)=1}} \lambda_{f}(p) \lambda_{g}(p) \frac{\lambda_{f}(p) \lambda_{g}(p)}{4}\right)^{2}-8 \sum_{\substack{p \leq \sqrt{x},(p, N)=1}} 1 \\
& =\frac{1}{32}\left(\sum_{\substack{p \leq \sqrt{x},(p, N)=1}} \lambda_{f}(p)^{2} \lambda_{g}(p)^{2}\right)^{2}+O\left(\frac{\sqrt{x}}{\log x}\right) \\
& \gg \frac{x}{\log ^{2} x} .
\end{aligned}
$$

This completes the proof of the lemma.

We are now in a position to complete the proof of Theorem 1 ,

Proof. Assume that $\lambda_{f}\left(p^{\alpha}\right) \lambda_{g}\left(p^{\alpha}\right) \geq 0$ for all $p^{\alpha} \leq x$ with $\alpha \leq 2$. By Lemma 12, we see that

$$
\sum_{\substack{n \leq x / 2,(n, N)=1, n \text { square-free }}} \lambda_{f}(n) \lambda_{g}(n) \log ^{2}(x / n) \gg \sum_{\substack{n \leq x / 2,(n, N)=1, n \text { square-free }}} \lambda_{f}(n) \lambda_{g}(n) \gg \frac{x}{\log ^{2} x}
$$

provided $x \geq \exp \left(c \log ^{2}(\sqrt{\mathfrak{q}(f)}+\sqrt{\mathfrak{q}(g)})\right)$, where $c>0, \mathfrak{q}(f), \mathfrak{q}(g)$ are as in Lemma 12. Now comparing (10) and (13), for any $\epsilon>0$, we have

$$
x \ll_{\epsilon} \max \left\{\exp \left(c \log ^{2}(\sqrt{\mathfrak{q}(f)}+\sqrt{\mathfrak{q}(g)}),\left[N^{2}\left(1+\frac{k_{2}-k_{1}}{2}\right)\left(\frac{k_{1}+k_{2}}{2}\right)\right]^{1+\epsilon}\right\},\right.
$$

where $c, \mathfrak{q}(f), \mathfrak{q}(g)$ are as before. Here we have used Lemma 4 of Choie and Kohnen [5]. This completes the proof of Theorem 1 .

\section{PRoOf OF THE THEOREM 2}

We now state a Lemma which we shall use to prove Theorem 2 .

Lemma 13. Let $\left\{a_{n}\right\}_{n \in \mathbb{N}}$ and $\left\{b_{m}\right\}_{m \in \mathbb{N}}$ be two sequences of real numbers such that

(1) $a_{n}=O\left(n^{\alpha_{1}}\right), \quad b_{m}=O\left(m^{\alpha_{2}}\right)$,

(2) $\sum_{n, m \leq x} a_{n} b_{m} \ll x^{\beta}$,

(3) $\sum_{n, m \leq x} a_{n}^{2} b_{m}^{2}=c x+O\left(x^{\gamma}\right)$,

where $\alpha_{1}, \alpha_{2}, \beta, \gamma \geq 0$ and $c>0$ such that $\max \left\{\alpha_{1}+\alpha_{2}+\beta, \gamma\right\}<1$. Then for any $r$ satisfying

$$
\max \left\{\alpha_{1}+\alpha_{2}+\beta, \gamma\right\}<r<1,
$$

there exists a sign change among the elements of the sequence $\left\{a_{n} b_{m}\right\}_{n, m \in \mathbb{N}}$ for $n, m \in\left[x, x+x^{r}\right]$. Consequently, for sufficiently large $x$, the number of sign changes among the elements of the sequence $\left\{a_{n} b_{m}\right\}_{n, m \in \mathbb{N}}$ with $n, m \leq x$ are $\gg x^{1-r}$. 
Proof. Suppose that for any $r$ satisfying

$$
\max \left\{\alpha_{1}+\alpha_{2}+\beta, \gamma\right\}<r<1
$$

the elements of the sequence $\left\{a_{n} b_{m}\right\}_{n, m \in \mathbb{N}}$ have same signs in $\left[x, x+x^{r}\right]$. This implies that

$$
x^{r} \ll \sum_{x \leq n, m \leq x+x^{r}} a_{n}^{2} b_{m}^{2} \ll x^{\alpha_{1}+\alpha_{2}} \sum_{x \leq n, m \leq x+x^{r}} a_{n} b_{m} \ll x^{\alpha_{1}+\alpha_{2}+\beta},
$$

which is a contradiction. This completes the proof of the Lemma.

Lemma 13 can be thought of as a generalization of a Lemma of Meher and Ram Murty (see [23. Theorem 1.1]) when $b_{1}=1$ and $b_{m}=0$ for all $m>1$. We are now in a position to prove Theorem 2 .

Proof. In order to apply Lemma 13, we need to verify the following conditions for the elements of the sequence $\left\{\lambda_{f}(n) \lambda_{g}\left(n^{2}\right)\right\}_{n \in \mathbb{N}}$. Note that

(1) Ramanujan-Deligne bound implies that

$$
\lambda_{f}(n) \lambda_{g}\left(n^{2}\right)=O_{\epsilon}\left(n^{\epsilon}\right)
$$

for all $n \in \mathbb{N}$.

(2) By a recent work of Lü [21, Theorem 1.2(2)] (see also Kumari and Ram Murty [18]), one has

$$
\sum_{n \leq x} \lambda_{f}(n) \lambda_{g}\left(n^{2}\right) \ll x^{5 / 7}(\log x)^{-\theta / 2}
$$

where $\theta=1-\frac{8}{3 \pi}=0.1512 \ldots$

(3) In the same paper, Lü (see [21, Lemma 2.3(ii)] as well as Kumari and Ram Murty [18]) also proved that

$$
\sum_{n \leq x} \lambda_{f}(n)^{2} \lambda_{g}\left(n^{2}\right)^{2}=c x+O\left(x^{\frac{17}{18}+\epsilon}\right)
$$

where $c>0$.

Theorem 2 now follows from Lemma 13 by choosing $a_{n}=\lambda_{f}(n)$ and $b_{m}:=\lambda_{g}\left(m^{2}\right)$ for all $m, n \in \mathbb{N}$ and considering the sequence $\left\{a_{n} b_{n}\right\}_{n \in \mathbb{N}}$.

\section{Proof of the theorem 3}

Using equation (4), one can write

$$
\lambda_{f}(p)=2 \cos \alpha_{p} \text { and } \lambda_{g}(p)=2 \cos \beta_{p}
$$


with $0 \leq \alpha_{p}, \beta_{p} \leq \pi$. Using the Hecke relation (3) for any prime $\left(p, N_{1} N_{2}\right)=1$, one has

$$
\lambda_{f}\left(p^{m}\right)= \begin{cases}(-1)^{m}(m+1) & \text { if } \alpha_{p}=\pi \\ m+1 & \text { if } \alpha_{p}=0 \\ \frac{\sin (m+1) \alpha_{p}}{\sin \alpha_{p}} & \text { if } 0<\alpha_{p}<\pi\end{cases}
$$

and

$$
\lambda_{g}\left(p^{m}\right)= \begin{cases}(-1)^{m}(m+1) & \text { if } \beta_{p}=\pi \\ m+1 & \text { if } \beta_{p}=0 \\ \frac{\sin (m+1) \beta_{p}}{\sin \beta_{p}} & \text { if } 0<\beta_{p}<\pi .\end{cases}
$$

Theorem 3 now follows from the following four cases.

Case (1): When $\alpha_{p}=0$ or $\pi$ and $\beta_{p}=0$ or $\pi$, then by the equation (14) and equation (15), we see that

$$
\left\{m \in \mathbb{N} \mid a_{f}\left(p^{m}\right) a_{g}\left(p^{m}\right) \neq 0\right\}=\mathbb{N} .
$$

In this case all elements of the sequence $\left\{a_{f}\left(p^{m}\right) a_{g}\left(p^{m}\right)\right\}_{m \in \mathbb{N}}$ are non-zero.

Case (2): Suppose that at least one of $\alpha_{p}, \beta_{p}$, say $\alpha_{p}=0$ or $\pi$ and $\beta_{p} \in(0, \pi)$. If $\beta_{p} / \pi \notin \mathbb{Q}$, there is nothing to prove. Now if $\beta_{p} / \pi=\frac{r}{s}$ with $(r, s)=1$, then we have

$$
\#\left\{m \leq x \mid a_{f}\left(p^{m}\right) a_{g}\left(p^{m}\right) \neq 0\right\}=\#\left\{m \leq x \mid a_{g}\left(p^{m}\right) \neq 0\right\}=[x]-\left[\frac{x}{s}\right] .
$$

Hence the set $\left\{m \mid a_{f}\left(p^{m}\right) a_{g}\left(p^{m}\right) \neq 0\right\}$ has postive density.

Case (3): Suppose that $\alpha_{p}=\beta_{p} \in(0, \pi)$, i.e. $\alpha_{p} / \pi=\beta_{p} / \pi \in(0,1)$. If $\alpha_{p} / \pi \notin \mathbb{Q}$, then $a_{f}\left(p^{m}\right) a_{g}\left(p^{m}\right) \neq 0$ for all $m \in \mathbb{N}$ as $\sin m \alpha_{p} \neq 0$ for all $m \in \mathbb{N}$. If $\alpha_{p} / \pi \in \mathbb{Q}$, say $\alpha_{p} / \pi=\frac{r}{s}$, where $r, s \in \mathbb{N}$ with $(r, s)=1$, then we have $\sin m \alpha_{p}=0$ if and only if $m$ is an integer multiple of $s$ and hence

$$
\#\left\{m \leq x: a_{f}\left(p^{m}\right) a_{g}\left(p^{m}\right) \neq 0\right\}=[x]-\left[\frac{x}{s}\right] .
$$

Hence the set in (2) has positive density.

Case (4): Assume that $\alpha_{p}, \beta_{p} \in(0, \pi)$ with $\alpha_{p} \neq \beta_{p}$. If both $\alpha_{p} / \pi, \beta_{p} / \pi \notin \mathbb{Q}$, then there is nothing to prove. Next suppose that one of them, say $\alpha_{p} / \pi \in \mathbb{Q}$ with $\alpha_{p} / \pi=\frac{r}{s}$ with $(r, s)=1$ and $\beta_{p} / \pi \notin \mathbb{Q}$. Then we have

$$
\#\left\{m \leq x: a_{f}\left(p^{m}\right) a_{g}\left(p^{m}\right) \neq 0\right\}=\#\left\{m \leq x: a_{f}\left(p^{m}\right) \neq 0\right\}=[x]-\left[\frac{x}{s}\right] .
$$

Hence the set in (2) has positive density.

Now let both $\alpha_{p} / \pi, \beta_{p} / \pi \in \mathbb{Q}$. If $\alpha_{p} / \pi=\frac{r_{1}}{s_{1}}$ and $\beta_{p} / \pi=\frac{r_{2}}{s_{2}}$ with $\left(r_{i}, s_{i}\right)=1$, for $1 \leq i \leq 2$, then

$$
\#\left\{m \leq x: a_{f}\left(p^{m}\right) a_{g}\left(p^{m}\right) \neq 0\right\}=\#\left[\left\{m \leq x: a_{f}\left(p^{m}\right) \neq 0\right\} \cap\left\{m \leq x: a_{g}\left(p^{m}\right) \neq 0\right\}\right] .
$$


Note that both $s_{1}$ and $s_{2}$ can not be 2 as otherwise $\alpha_{p}=\beta_{p}$. Since

$$
\begin{aligned}
\#\left\{m \leq x: a_{f}\left(p^{m}\right) a_{g}\left(p^{m}\right)=0\right\} & =\#\left[\left\{m \leq x: a_{f}\left(p^{m}\right)=0\right\} \cup\left\{m \leq x: a_{g}\left(p^{m}\right)=0\right\}\right] \\
& \leq\left[\frac{x}{s_{1}}\right]+\left[\frac{x}{s_{2}}\right],
\end{aligned}
$$

the set in (2) has positive density. This completes the proof of Theorem 3 ,

\section{Proof of Theorem 4}

Using Lemma 6, we see that for any $x \geq 2$ and $0<\delta<1 / 2$

$$
\#\left\{p \leq x: a_{f}\left(p^{m}\right)=0 \text { for some } m \geq 1\right\} \ll_{f, \delta} \frac{x}{(\log x)^{1+\delta}},
$$

where the implied constant depends only on $f$ and $\delta$. We have the same estimate for the form $g$ as well. Therefore for any $x \geq 2$ and $0<\delta<1 / 2$, we have

$$
\#\left\{p \leq x: a_{f}\left(p^{m}\right) a_{g}\left(p^{m^{\prime}}\right)=0 \text { for some } m, m^{\prime} \geq 1\right\} \ll_{f, g, \delta} \frac{x}{(\log x)^{1+\delta}},
$$

where the implied constant depends on $f, g$ and $\delta$. Hence

$$
\begin{aligned}
& \#\left\{p \leq x: a_{f}\left(p^{m}\right) a_{g}\left(p^{m^{\prime}}\right) \neq 0 \text { for all } m, m^{\prime} \geq 1\right\} \\
& =\pi(x)-\#\left\{p \leq x: a_{f}\left(p^{m}\right) a_{g}\left(p^{m^{\prime}}\right)=0 \text { for some } m, m^{\prime} \geq 1\right\},
\end{aligned}
$$

where $\pi(x)$ denotes the number of primes up to $x$. Now using prime number theorem as well as the identity (16), we have

$$
\#\left\{p \leq x: a_{f}\left(p^{m}\right) a_{g}\left(p^{m^{\prime}}\right) \neq 0 \text { for all } m, m^{\prime} \geq 1\right\} \sim \frac{x}{\log x} .
$$

Hence the set

$$
\left\{p \in \mathcal{P}: a_{f}\left(p^{m}\right) a_{g}\left(p^{m^{\prime}}\right) \neq 0 \text { for any integers } m, m^{\prime} \geq 1\right\}
$$

has natural density 1.

\section{Proof of theOREM 5}

We keep the notations in this section as in section 5. To prove Theorem 5, we start by proving the following Proposition.

Proposition 14. Let

$$
f(z)=\sum_{n=1}^{\infty} a_{f}(n) q^{n} \in S_{k_{1}}\left(N_{1}\right) \quad \text { and } \quad g(z)=\sum_{n=1}^{\infty} a_{g}(n) q^{n} \in S_{k_{2}}\left(N_{2}\right)
$$

be two distinct normalized Hecke eigenforms. Then for any prime $p$ with $\left(p, N_{1} N_{2}\right)=1$, there exists an integer $m$ with $1 \leq m \leq 4$ such that $a_{f}\left(p^{m}\right) a_{g}\left(p^{m}\right) \neq 0$. 
Proof. Note that $a_{f}\left(p^{m}\right) a_{g}\left(p^{m}\right) \neq 0$ is equivalent to $\sin (m+1) \alpha_{p} \sin (m+1) \beta_{p} \neq 0$. If $a_{f}(p) a_{g}(p) \neq$ 0 , then we are done. Now suppose $a_{f}(p) a_{g}(p)=0$, then either $a_{f}(p)=0$ or $a_{g}(p)=0$.

Case (1): If $a_{f}(p)=0=a_{g}(p)$, then $\alpha_{p}=\beta_{p}=\pi / 2$. Hence we have

$$
a_{f}\left(p^{2}\right) a_{g}\left(p^{2}\right)=p^{k_{1}+k_{2}-2} \neq 0 .
$$

Case (2): Suppose that at least one of $a_{f}(p), a_{g}(p) \neq 0$. Without loss of generality assume that $a_{f}(p)=0$ and $a_{g}(p) \neq 0$, then $\alpha_{p}=\pi / 2$ and $\beta_{p} \neq \pi / 2$. Now if $\beta_{p}=0$ or $\pi$, then $a_{g}\left(p^{2}\right)=3 p^{k_{2}-1}$. Hence we have

$$
a_{f}\left(p^{2}\right) a_{g}\left(p^{2}\right)=-3 p^{k_{1}+k_{2}-2} \neq 0
$$

If $\beta_{p} \notin\{0, \pi / 2, \pi\}$, then this implies that $a_{g}\left(p^{2}\right)=p^{\left(k_{2}-1\right)} \frac{\sin 3 \beta_{p}}{\sin \beta_{p}}$. Now if $a_{f}\left(p^{2}\right) a_{g}\left(p^{2}\right)=0$, then $\beta_{p} \in\{\pi / 3,2 \pi / 3\}$ as $0<\beta_{p}<\pi$. Then we have

$$
\frac{a_{f}\left(p^{4}\right) a_{g}\left(p^{4}\right)}{p^{2\left(k_{1}+k_{2}-2\right)}}=\frac{2}{\sqrt{3}} \sin \frac{5 \pi}{2} \sin \frac{5 \pi}{3} \quad \text { or } \quad \frac{a_{f}\left(p^{4}\right) a_{g}\left(p^{4}\right)}{p^{2\left(k_{1}+k_{2}-2\right)}}=\frac{2}{\sqrt{3}} \sin \frac{5 \pi}{2} \sin \frac{10 \pi}{3} .
$$

Since neither $\sin (5 \pi / 2) \sin (5 \pi / 3)$ nor $\sin (5 \pi / 2) \sin (10 \pi / 3)$ is equal to zero, this completes the proof of Proposition 14 .

Proof. We now complete the proof of the first part of Theorem 5 by showing the existence of a prime $p \leq 2 \log N$ with $(p, N)=1$ and then using Proposition 14. We know by a theorem of Rosser and Schoenfeld (see [30, p. 70]) that

$$
\sum_{p \leq x} \log p>0.73 x \quad \text { for all } x \geq 41 .
$$

Using this, one can easily check that

$$
\sum_{p \leq x} \log p>\frac{x}{2} \quad \text { for all } x \geq 5
$$

Now consider the following product

$$
\prod_{p \leq 2 \log N} p=\exp \left(\sum_{p \leq 2 \log N} \log p\right)>N
$$

which confirms the existence of such a prime. Proof of the second part of Theorem 5 follows immediately by applying Proposition 14 . 


\section{8. $\mathfrak{B}$-FREE NUMBERS AND SIMULTANEOUS NON-VANISHING IN SHORT INTERVALS}

In this section, we first list certain properties of $\mathfrak{B}$-free numbers and their distribution in short intervals to derive simultaneous non-vanishing of Hecke eigenvalues. Erdös [8] introduced the notion of $\mathfrak{B}$-free numbers and showed the existence of these numbers in short intervals.

Definition 1. Let us assume that

$$
\mathfrak{B}:=\left\{b_{1}, b_{2}, \ldots\right\} \subset \mathbb{N}
$$

be such that

$$
\left(b_{i}, b_{j}\right)=1 \text { for } i \neq j \quad \text { and } \quad \sum_{i \geq 1} \frac{1}{b_{i}}<\infty .
$$

One says that a number $n \in \mathbb{N}$ is $\mathfrak{B}$-free if it is not divisible by any element of the set $\mathfrak{B}$.

The distribution of $\mathfrak{B}$-free numbers in short intervals has been studied by several mathematicians (see [3], [31], [34], [35], [37]). Balog and Ono [4] were first to use $\mathfrak{B}$-free numbers to study non-vanishing of Hecke eigenvalues.

For a non-CM cusp form $f \in S_{k}(N)$ with Fourier coefficients $\left\{a_{f}(n)\right\}_{n \in \mathbb{N}}$, Serre (see [33, page 383]) defined the function

$$
i_{f}(n):=\max \left\{m \in \mathbb{N} \mid a_{f}(n+j)=0 \text { for all } 0<j \leq m\right\}
$$

which is now known as gap function. Alkan and Zaharescu [1] proved that

$$
i_{\Delta}(n) \ll_{\Delta} n^{1 / 4+\epsilon}
$$

for Ramanujan $\Delta$-function. Kowalski, Robert and $\mathrm{Wu}$ [17], using distribution of $\mathfrak{B}$-free numbers in short intervals showed that

$$
i_{f}(n) \ll_{f} n^{7 / 17+\epsilon}
$$

where $f \in S_{k}^{\text {new }}(N)$ is any normalized Hecke eigenform. Recently, Das and Ganguly [6] showed that

$$
i_{f}(n) \ll_{f} n^{1 / 4+\epsilon}
$$

for any $f \in S_{k}(1)$.

In this article, we will study simultaneous non-vanishing of Hecke eigenvalues using $\mathfrak{B}-$ free numbers. This question was first considered by Kumari and Ram Murty [18]. We now introduce the set of $\mathfrak{B}$-free numbers as constructed by Kowalski, Robert and $\mathrm{Wu}$ [17]. These numbers will play an important role in our work.

Let $\mathfrak{P}$ be a subset of $\mathcal{P}$ such that

$$
\#(\mathfrak{P} \cap[1, x]) \ll \frac{x^{\rho}}{(\log x)^{\eta_{\rho}}}
$$


where $\rho \in[0,1]$ and $\eta_{\rho}$ 's are real numbers with $\eta_{1}>1$. Let us define

$$
\mathfrak{B}_{\mathfrak{P}}:=\mathfrak{P} \cup\left\{p^{2} \mid p \in \mathcal{P}-\mathfrak{P}\right\} .
$$

Write $\mathfrak{B}_{\mathfrak{P}}=\left\{b_{i} \mid i \in \mathbb{N}\right\}$. Note that $\left(b_{i}, b_{j}\right)=1$ for all $b_{i}, b_{j} \in \mathfrak{B}_{\mathfrak{P}}$ with $b_{i} \neq b_{j}$. To show $\sum_{i \in \mathbb{N}} \frac{1}{b_{i}}<\infty$, it is enough to show that $\sum_{p \in \mathfrak{P}} \frac{1}{p}<\infty$. Applying equation (17) and partial summation formula, one has

$$
\sum_{\substack{p \leq x, p \in \mathfrak{P}}} \frac{1}{p}=\frac{1}{x} \sum_{\substack{p \leq x, p \in \mathfrak{P}}} 1+\int_{2}^{x} \frac{1}{t^{2}}\left(\sum_{\substack{p \leq t, p \in \mathfrak{P}}} 1\right) d t \ll_{\mathfrak{P}} \frac{x^{\rho-1}}{(\log x)^{\eta_{\rho}}}+\int_{2}^{x} \frac{t^{\rho-2}}{(\log t)^{\eta_{\rho}}} d t \ll_{\mathfrak{P}} 1 .
$$

With these notations, Kowalski, Robert and Wu (see Corollary 10 of [17]) proved the following Theorem.

Theorem 15 (Kowalski, Robert and $\mathrm{Wu}$ ). For any $\epsilon>0, x \geq x_{0}(\mathfrak{P}, \epsilon)$ and $y \geq x^{\theta(\rho)+\epsilon}$, we have

$$
\#\left\{x<n \leq x+y \mid n \text { is } \mathfrak{B}_{\mathfrak{P}-} \text {-free }\right\} \gg_{\mathfrak{P}, \epsilon} y,
$$

where

$$
\theta(\rho):= \begin{cases}\frac{1}{4} & \text { if } 0 \leq \rho \leq \frac{1}{3} \\ \frac{10 \rho}{19 \rho+7} & \text { if } \frac{1}{3}<\rho \leq \frac{9}{17} \\ \frac{3 \rho}{4 \rho+3} & \text { if } \frac{9}{17}<\rho \leq \frac{15}{28} \\ \frac{5}{16} & \text { if } \frac{15}{28}<\rho \leq \frac{5}{8} \\ \frac{22 \rho}{24 \rho+29} & \text { if } \frac{5}{8}<\rho \leq \frac{9}{10} \\ \frac{7 \rho}{9 \rho+8} & \text { if } \frac{9}{10}<\rho \leq 1 .\end{cases}
$$

We now study simultaneous non-vanishing in short arithmetic progression using the distribution of $\mathfrak{B}$-free numbers. The question of the distribution of $\mathfrak{B}$-free numbers in short arithmetic progression was first considered by Alkan and Zaharescu [2]. In this direction, $\mathrm{Wu}$ and Zhai (see Proposition 4.1 of [36]) have the following result about distribution of $\mathfrak{B}$-free numbers in short arithmetic progression.

Theorem 16 (Wu and Zhai). Let $\mathfrak{B}_{\mathfrak{P}}$ be as in (18). For any $\epsilon>0, x \geq x_{0}(\mathfrak{P}, \epsilon), y \geq x^{\psi(\rho)+\epsilon}$ and $1 \leq a \leq q \leq x^{\epsilon}$ with $(a, q)=1$, one has

$$
\#\left\{x<n \leq x+y: n \text { is } \mathfrak{B}_{\mathfrak{P}} \text {-free and } n \equiv a(\bmod q)\right\} \gg_{\mathfrak{P}, \epsilon} y / q
$$

where

$$
\psi(\rho):= \begin{cases}\frac{29 \rho}{46 \rho+19} & \text { if } \frac{190}{323}<\rho \leq \frac{166}{173} \\ \frac{17 \rho}{26 \rho+12} & \text { if } \frac{166}{173}<\rho \leq 1\end{cases}
$$

Using above results, we now have the following non-vanishing Theorem for certain multiplicative function. 
Theorem 17. Let $f: \mathbb{N} \rightarrow \mathbb{C}$ be a multiplicative function and let $N \geq 1$ be a positive integer. Define

$$
\mathfrak{P}_{f, N}:=\{p \in \mathcal{P} \mid f(p)=0\} \cup\{p \in \mathcal{P}|p| N\} .
$$

Also assume that $\mathfrak{P}_{f, N}$ satisfies condition (17). Then

(1) For any $\epsilon>0, x \geq x_{0}\left(\mathfrak{P}_{f, N}, \epsilon\right)$ and $y \geq x^{\theta(\rho)+\epsilon}$, we have

$$
\#\{x<n \leq x+y \mid(n, N)=1, n \text { square-free and } f(n) \neq 0\} \gg_{\mathfrak{P}_{f, N}, \epsilon} y,
$$

where $\theta(\rho)$ is as in (19).

(2) For any $\epsilon>0, x \geq x_{0}\left(\mathfrak{P}_{f, N}, \epsilon\right), y \geq x^{\psi(\rho)+\epsilon}$ and $1 \leq a \leq q \leq x^{\epsilon}$ with $(a, q)=1$, we have

$\#\{x<n \leq x+y:(n, N)=1, n$ square-free, $n \equiv a(\bmod q)$ and $f(n) \neq 0\} \gg_{\mathfrak{P}_{f, N}, \epsilon} y / q$,

where $\psi(\rho)$ is as in (20).

Proof. Define

$$
\mathfrak{B}_{\mathfrak{P}_{f, N}}:=\mathfrak{P}_{f, N} \cup\left\{p^{2} \mid p \in \mathcal{P}-\mathfrak{P}_{f, N}\right\} .
$$

Then first part of Theorem 17 now follows from Theorem 15, Applying Theorem 16, we get the second part of Theorem 17 .

As an immediate corollary, we have

Corollary 18. Let $E_{1} / \mathbb{Q}$ and $E_{2} / \mathbb{Q}$ be two non-CM elliptic curves which have the same conductor $N$. Let

$$
L\left(E_{i}, s\right)=\sum_{n=1}^{\infty} a_{E_{i}}(n) n^{-s}, \quad i=1,2
$$

be their Hasse-Weil L-functions. If $f_{E_{i}}(z)=\sum_{n=1}^{\infty} a_{E_{i}}(n) q^{n}$ for $i=1,2$ are the associated weight two newforms, then

(1) for any $\epsilon>0$ and $y \geq x^{33 / 94+\epsilon}$, we have

$$
\#\left\{x<n<x+y \mid n \text { is square-free and } a_{E_{1}}(n) a_{E_{2}}(n) \neq 0\right\} \gg_{E_{1}, E_{2}, \epsilon} y .
$$

(2) For any $\epsilon>0, x \geq x_{0}\left(E_{1}, E_{2}, \epsilon\right), y \geq x^{87 / 214+\epsilon}$ and $1 \leq a \leq q \leq x^{\epsilon}$ with $(a, q)=1$, we have

$$
\begin{aligned}
\#\{x<n \leq x+y \quad \mid & \left.(n, N)=1, n \text { is square-free and } n \equiv a(\bmod q) \text { and } a_{E_{1}}(n) a_{E_{2}}(n) \neq 0\right\} \\
& \gg_{E_{1}, E_{2}, \epsilon} y / q .
\end{aligned}
$$

Proof. Let $\pi_{E}(x)$ be the number of supersingular primes up to $x$ for a non-CM elliptic curve $E / \mathbb{Q}$. By the work of Elkies [7], we have

$$
\#\left\{p \leq x: a_{E}(p)=0\right\} \ll_{E} x^{3 / 4} .
$$

Considering $f(n):=a_{E_{1}}(n) a_{E_{2}}(n)$, one easily sees that $\mathfrak{P}_{f, N}$ satisfies condition (17) with $\rho=$ $3 / 4$ and $\eta_{\rho}=0$. Now by using Theorem 17 , we get the Corollary. 
Kumari and Ram Murty have proved similar results for non-CM cusp forms which are newforms and normalized Hecke eigenforms of weight $k>2$.

As a second corollary, we have the following simultaneous non-vanishing result for coefficients of symmetric power $L$-functions in short intervals.

To state the corollary, we need to introduce few more notations. Let $f \in S_{k}^{n e w}(N)$ be a normalized Hecke eigenform with Fourier coefficients $\left\{a_{f}(n)\right\}_{n \in \mathbb{N}}$. Set $\lambda_{f}(n)=a_{f}(n) / n^{(k-1) / 2}$ and suppose that for $p \nmid N, \alpha_{f, p}, \beta_{f, p}$ are the Satake $p$-parameter of $f$. Then the un-ramified $m$-th symmetric power $L$-function of $f$ is defined as follows:

$$
L_{u n r}\left(\operatorname{sym}^{m} f, s\right):=\prod_{p \nmid N} \prod_{0 \leq j \leq m}\left(1-\alpha_{f, p}^{j} \beta_{f, p}^{m-j} p^{-s}\right)^{-1}=: \sum_{n \geq 1} \lambda_{f}^{(m)}(n) n^{-s} .
$$

We now have the following corollary.

Corollary 19. Let $f \in S_{k_{1}}^{n e w}\left(N_{1}\right)$ and $g \in S_{k_{2}}^{\text {new }}\left(N_{2}\right)$ be normalized non-CM Hecke eigenforms. Let $N:=\operatorname{lcm}\left[N_{1}, N_{2}\right]$. Then

(1) for any $\epsilon>0, x \geq x_{0}(f, g, \epsilon)$ and $y \geq x^{7 / 17+\epsilon}$, we have

$$
\#\left\{x<n \leq x+y \mid n \text { is square-free and } \lambda_{f}^{(m)}(n) \lambda_{g}^{(m)}(n) \neq 0\right\} \gg_{f, g, m, \epsilon} y .
$$

(2) For any $\epsilon>0, x \geq x_{0}(f, g, \epsilon), y \geq x^{17 / 38+\epsilon}$ and $1 \leq a \leq q \leq x^{\epsilon}$ with $(a, q)=1$, we have $\#\left\{x<n \leq x+y \mid(n, N)=1, n\right.$ square-free, $n \equiv a(\bmod q)$ and $\left.\lambda_{f}^{(m)}(n) \lambda_{g}^{(m)}(n) \neq 0\right\} \gg_{f, g, \epsilon} y / q$.

Proof. Let

$$
\mathfrak{P}_{f, g, m}:=\left\{p \in \mathcal{P}|p| N \text { or } \lambda_{f}^{(m)}(p) \lambda_{g}^{(m)}(p)=0\right\}
$$

Since $\lambda_{f}^{(m)}(p)=\lambda_{f}\left(p^{m}\right)$, using Lemma 6, we see that $\mathfrak{P}_{f, g, m}$ satisfies condition (17). Note that $f(n):=\lambda_{f}^{(m)}(n) \lambda_{g}^{(m)}(n)$ is a multiplicative function and hence we can apply Theorem 17, to complete the proof of Corollary 19.

Remark 8.1. Note that Corollary 19 implies simultaneous non-vanishing of Hecke eigenvalues in sparse sequences. More precisely, let $f \in S_{k_{1}}^{n e w}\left(N_{1}\right)$ and $g \in S_{k_{2}}^{n e w}\left(N_{2}\right)$ be normalized non-CM Hecke eigenforms. Also let $N:=\operatorname{lcm}\left[N_{1}, N_{2}\right]$. Then

(1) for any $\epsilon>0, x \geq x_{0}(f, g, \epsilon)$ and $y \geq x^{7 / 17+\epsilon}$, we have $\#\left\{x<n \leq x+y:(n, N)=1, n\right.$ is square-free and $\left.\lambda_{f}\left(n^{m}\right) \lambda_{g}\left(n^{m}\right) \neq 0\right\} \gg_{f, g, m, \epsilon} y$.

(2) For any $\epsilon>0, x \geq x_{0}(f, g, \epsilon), y \geq x^{17 / 38+\epsilon}$ and $1 \leq a \leq q \leq x^{\epsilon}$ with $(a, q)=1$, we have $\#\left\{x<n \leq x+y:(n, N)=1, n\right.$ square-free, $n \equiv a(\bmod q)$ and $\left.\lambda_{f}\left(n^{m}\right) \lambda_{g}\left(n^{m}\right) \neq 0\right\} \gg_{f, g, \epsilon} y / q$.

Acknowledgments: The authors would like to thank Jyoti Sengupta for bringing to their notice the paper of Lau-Liu-Wu [19]. The authors would also like to thank Purusottam Rath for going through an earlier version of the paper. 


\section{REFERENCES}

[1] E. Alkan and A. Zaharescu, Nonvanishing of the Ramanujan tau function in short intervals, Int. J. Number Theory 1 (2005), no. 1, 45-51.

[2] E. Alkan and A. Zaharescu, $\mathfrak{B}$-free numbers in short arithmetic progressions, J. Number Theory 113 (2005), 226243.

[3] G. Bantle and F. Grupp, On a problem of Erdös and Szemerédi, J. Number Theory 22 (1986), 280-288.

[4] A. Balog and K. Ono, The Chebotarev density theorem in short intervals and some questions of Serre, J. Number Theory 91 (2001), 356-371.

[5] Y. Choie and W. Kohnen, The first sign change of Fourier coefficients of cusp forms, Amer. J. Math. 131 (2009), no. 2, 517-543.

[6] S. Das and S. Ganguly, Gaps between non-zero Fourier coefficients of cusp forms, Proc. Amer. Math. Soc. 142 (2014), 3747-3755.

[7] N. Elkies, Distribution of supersingular primes, In Journées Arithmétiques (1989), Astérisque, (1992), $127-132$.

[8] P. Erdös, On the difference of consecutive terms of sequences defined by divisibility properties, Acta Arith. 12 (1966/1967), 175-182.

[9] S. Gun, W. Kohnen and P. Rath, Simultaneous sign change of Fourier-coefficients of two cusp forms, Arch. Math. (Basel) 105 (2015), no. 5, 413-424.

[10] A. E. Ingham, The distribution of prime numbers, Cambridge University Press, Cambridge, 1990.

[11] H. Iwaniec and E. Kowalski, Analytic Number Theory, American Mathematical Society, 2004.

[12] H. Iwaniec, W. Kohnen and J. Sengupta, The first negative Hecke eigenvalue, Int. J. Number Theory 3 (2007), 355-363.

[13] W. Kohnen, On Hecke eigenvalues of newforms, Math. Ann. 329 (2004), no. 4, 623-628.

[14] W. Kohnen and J. Sengupta, Signs of Fourier coefficients of two cusp forms of different weights, Proc. Amer. Math. Soc. 137 (2009), no. 11, 3563-3567.

[15] E. Kowalski, Y. K. Lau, K. Soundararajan and J. Wu, On modular signs, Math. Proc. Cambridge Philos. Soc. 149 (2010), no. 3, 389-411.

[16] E. Kowalski, P. Michel and J. Vanderkam, Rankin-Selberg L-functions in the level aspect, Duke Mathematical Journal 114 (2002), 123-191.

[17] E. Kowalski, O. Robert and J. Wu, Small gaps in coefficients of L-functions and $\mathfrak{B}$-free numbers in short intervals, Rev. Mat. Iberoamericana, 23 (2007), no. 1, 281-326.

[18] M. Kumari and M.R. Murty, Simultaneous non-vanishing and sign changes of Fourier coefficients of modular forms, submitted.

[19] Y-K. Lau, J. Liu and J. Wu, Sign changes of the coefficients of automorphic L-functions, Number Theory: Arithmetic in Shangri-La, edited by S. Kanemitsu, H. Li, and J. Liu, 141-181. Hackensack, NJ: World Scientific Publishing Co. Pvt. Ltd., 2013.

[20] W-C Winnie Li, L-series of Rankin type and their functional equations, Math. Ann. 244 (1979), $135-166$.

[21] G. Lü, Sums of absolute values of cusp form coefficients and their application, J. Number Theory, 139 (2014), $29-43$.

[22] K. Matomäki, On signs of Fourier coefficients of cusp forms, Math. Proc. Cambridge Philos. Soc. 152 (2012), no. 2, 207-222.

[23] J. Meher and M. R. Murty, Sign changes of Fourier coefficients of half-integral weight cusp forms, Int. J. Number Theory, 10 (2014), No. 4, 905-914.

[24] M. Ram Murty, Problems in analytic number theory, Graduate Texts in Mathematics 206, Springer-Verlag, New York, 2001. 
[25] M. R. Murty, Congruences between modular forms, Analytic Number Theory, Kyoto, (1996),309-320, London Math., Soc., Lecture Notes, Ser., 247, Cambridge University Press, Cambridge, (1997).

[26] M. R. Murty and V. K. Murty, Odd values of Fourier coefficients of certain modular forms, Int. J. Number Theory, 3 (2007), no. 3, 455-470.

[27] A.P. Ogg, On a convolution of L-series, Invent. Math. 7 (1969), 297-312.

[28] H. Rademacher, On the Phragmén-Lindelöf theorem and some applications, Math. Z. 72 (1959), $192-204$.

[29] R.A. Rankin, Contributions to the theory of Ramanujan's function $\tau(n)$ and similar arithmetical functions II. Proc. Cambridge Philos. Soc. 35 (1939), 357-372.

[30] J. B. Rosser and L. Schoenfeld, Approximate formulas for some functions of prime numbers, Illinois J. Math. 6 (1962), 64-94.

[31] P. Sargos, and J. Wu, Multiple exponential sums with monomials and their applications in number theory, Acta Math. Hungar. 87 (2000), 333-354.

[32] J. Sengupta, Distinguishing Hecke eigenvalues of primitive cusp forms, Acta Arith. 114 (2004), no. 1, $23-34$.

[33] J.P. Serre, Quelques applications du théoréme de densité de Chebotarev, Inst. Hautes Études Sci. Publ. Math. 54 (1981), 323-401.

[34] E. Szemerédi, On the difference of consecutive terms of sequences defined by divisibility properties. II, Acta Arith. 23 (1973), 359-361.

[35] J. Wu, Nombres $\mathfrak{B}$-libres dans les petits intervalles, Acta Arith. 65 (1993), 97-116.

[36] J. Wu and W. Zhai, Distribution of Hecke eigenvalues of newforms in short intervals, Q. J. Math., 64(2) (2013), 619-644.

[37] W. G. Zhai, Number of $\mathfrak{B}$-free numbers in short intervals, Chinese Sci. Bull. 45 (2000), 208-212.

(Sanoli Gun, Balesh Kumar and Biplab Paul) Institute of Mathematical Sciences, Homi BHABHA NATiOnAl Institute, C.I.T CAMPUs, TARAMANi, CHENNAi 600 113, INDiA.

E-mail address: sanolieimsc.res.in

E-mail address: baleshkeimsc.res.in

E-mail address: biplabpauleimsc.res.in 